\title{
Hans Lippershey (ou Lipperhey)
}

Opticien-verrier et fabricant de lentilles hollandais d'origine allemande, Hans Lippershey est généralement reconnu comme le premier à avoir décrit (à défaut d'avoir inventé) le concept de la longue-vue, dont dérivent ceux de la lunette astronomique et du télescope.

\section{Riad HAIDAR, haidar@onera.fr}

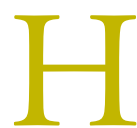

ans Lippershey (parfois orthographié Lipperhey), également appelé Jan Lippersheim, naît aux alentours de 1570 à Wesel, une ville membre de la puissante Hanse germanique (association de villes marchandes $\mathrm{d}^{\prime}$ Europe du Nord, très influente entre le $12^{\mathrm{e}}$ et le $17^{\mathrm{e}}$ siècles), et à ce titre particulièrement prospère. Notons que Wesel, située au bord du cours inférieur du Rhin, à la limite nord-ouest de la région de la Ruhr en actuelle Rhénaniedu-Nord - Westphalie, se trouve à $45 \mathrm{~km}$ à peine de la frontière néerlandaise.

On en sait peu sur l'enfance de Lippershey, mais on retrouve sa trace en 1594. À cette époque, il se marie et s'installe justement aux Pays-Bas, dans la ville de Middelbourg (Middelburg en néerlandais), située dans l'ancienne île de Walcheren sur le canal éponyme, dans la province de

\section{Le saviez-vous?}

Hans Lippershey n'a probablement pas été le premier à expérimenter la combinaison de lentilles pour créer des instruments optiques grossissants, et on ne saura probablement jamais qui est l'inventeur de la longue-vue (et donc du télescope). Qu'on en juge: certaines histoires parlent d'un dispositif télescopique «magique» au $16^{\mathrm{e}}$ siècle (donc antérieur à celui de Lippershey); quelques historiens attribuent à Giambattista della Porta de Naples la découverte des propriétés télescopiques des combinaisons de lentilles dès 1589; et puis, trois semaines à peine après la demande de brevet de Lippershey, Jacob Metius, maître-verrier renommé, installé à Alkmaar, une ville du nord des Pays-Bas, déclare être également l'inventeur de la longue-vue; plus tard encore, c'est au tour de Zacharias Janssen, opticien-lunetier à Middelbourg comme Lippershey, de réclamer la paternité de la longue-vue... Néanmoins, si la genèse de l'instrument reste inconnue, Lippershey est généralement considéré comme le premier à avoir décrit la longue-vue dans un document. Dans la version originale, Lippershey considère un système fait soit de deux lentilles convexes (donnant une image inversée), soit d'un objectif convexe et d'un oculaire concave (donnant une image bien orientée). Par ces combinaisons, sa longue-vue présente un grossissement $\times 3$.

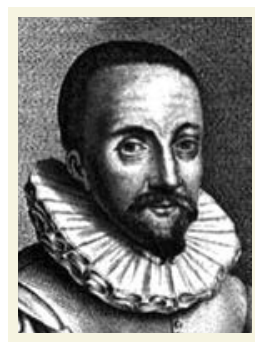

PRINCIPALES DATES

1570 - Naissance à Wesel (Rhénanie-du-Nord Westphalie, Allemagne)

1594 Apprenti-verrier

1608 Demande de brevet pour la longue-vue

1619 - Décès à Middelbourg (Zélande, Pays-Bas)

Portrait de Hans Lippershey

Zélande. Middelbourg est alors une ville florissante, surtout depuis que la puissante Anvers est tombée aux mains des espagnols en 1585 et qu'elle s'est vidée de ses protestants, qui constituaient une très large part de son élite commerciale et intellectuelle.

Lippershey apprend le métier de polisseur de lentilles, et devient un maître-verrier réputé. L'usinage du verre est alors une technique délicate venue de Venise et Florence, où elle est née au $13^{\mathrm{e}}$ siècle, qui s'est enrichie de nouveaux procédés dans les années 1590 (parmi lesquels probablement quelques idées sur la combinaison de lentilles) et s'est étendue aux Pays-Bas et en Allemagne.

À l'issue de son apprentissage, Lippershey ouvre une échoppe d'opticien lunetier et, en 1602, il est naturalisé citoyen de Zélande.

\section{Invention de la longue-vue?}

Pour les historiens, Hans Lippershey est le premier à avoir décrit, à défaut de l'avoir inventé, le concept de la longue-vue (i.e., un instrument d'optique monoculaire destiné à obtenir une vision rapprochée des objets éloignés), dont dérivent ceux de la lunette astronomique et du télescope.

Il est certes possible que Lippershey ait fait cette découverte par lui-même, mais il est plus probable qu'il l'ait reprise de son entourage. Selon une des légendes qui circulent, l'idée lui en serait venue en observant deux enfants qui jouaient avec des lentilles dans son échoppe, et qui remarquèrent qu'en la regardant à travers deux lentilles, une girouette sur une église voisine leur paraissait plus grande et plus lumineuse. Lippershey aurait alors refait lui-même l'expérience et réalisé tout le potentiel de l'instrument... Quoi qu'il en soit, plaçant un tube entre les lentilles, Lippershey rend le système plus maniable, le baptise «kijker», ce qui signifie littéralement «viseur» en néerlandais, et dépose une demande de brevet.

\section{Demande de brevet}

Le 25 septembre 1608, le gouverneur de Zélande adresse, à la demande de Lippershey, une lettre au stathouder Maurice de Nassau, gouverneur général des Provinces-Unies des 


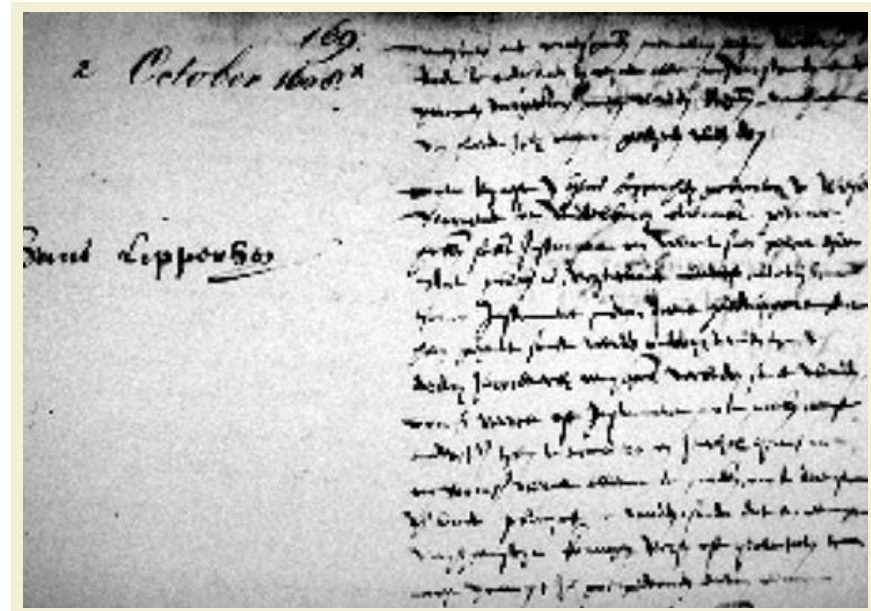

Demande de brevet de Lippershey, datant de septembre 1608.

Pays-Bas, où il est fait mention d'un «certain dispositif au moyen duquel toutes les choses à une très grande distance peuvent être vues comme si elles étaient à proximité» et qui semble être une nouvelle invention. Le 2 octobre 1608, Lippershey introduit une demande de brevet pour cet instrument - ou sollicite, à défaut, une pension annuelle, en échange de quoi il s'engage à ne pas vendre ses longues-vues aux rois étrangers. Sa demande de brevet n'aboutira finalement pas, car l'invention est jugée difficile à tenir secrète, mais le stathouder lui accorde une compensation financière conséquente.

\section{Dissémination de l'idée}

Comme pour confirmer l'intuition du stathouder sur la difficulté de garder le secret, la demande de brevet de Lippershey est mentionnée à la fin du rapport de la mission diplomatique du Royaume de Siam envoyée aux Pays-Bas par le roi Ekathotsarot. Ce rapport, rendu public à la fin de 1608, donne naissance à une rumeur persistante sur le kijker de Lippershey, une rumeur qui se propage irrésistiblement et suscite immédiatement des initiatives un peu partout en Europe. Citons Paolo Sarpi en Italie; Jacques Badovere en France; Thomas Harriot en Angleterre; et puis, enfin, Galilée... Galilée, dont le génie améliore l'instrument, le transforme en une lunette astronomique avec un grossissement $\times 20$. Avec sa lunette, il repère des montagnes et des cratères sur la lune, il comprend que la Voie Lactée est composée d'étoiles, et il découvre les quatre grandes lunes de Jupiter.

Étranger à cette formidable agitation scientifique, qu'il a involontairement initiée et à laquelle il ne participe pas, Hans Lippershey décède aux Pays-Bas en 1619. Il est enterré à Middelbourg le 29 septembre.

\section{RÉFÉRENCES}

[1] Robert Halleux, La science classique, Flammarion (1998)

[2] Albert Van Helden, The Invention of the Telescope, Philadelphia (1977)

\section{Caméra 3D ultra-flexible IDS présente des modèles des séries Ensenso $X$}

Aujourd ' hui, la vision 3D est encore plus simple, plus précise et plus souple: les nouvelles séries Ensenso $X$ constituent un système caméra 3D ultra-flexible, comprenant un projecteur d'une puissance LED $100 \mathrm{~W}$ et un switch GigE intégré, qui permet des réglages parfaitement sur mesure pour coller à vos besoins.

Les supports de fixation de la caméra sont disponibles dans une gamme de largeurs permettant l'implémentation des longueurs de base de différente taille. Vous pouvez aussi configurer l'angle de vue de la caméra et choisir entre des montures d'objectif variées $C$ pour la caméra et le projecteur. Cette souplesse autorise un large éventail de distances de travail jusqu'à peu près 7,5 mètres, vous permettant de capter des objets d'un volume de plusieurs mètres carrés, par exemple des palettes ou des salles entières.

La technologie FlexView2 utilisée dans les séries Ensenso $X$ améliore la précision $\mathrm{Z}$ jusqu'à un facteur

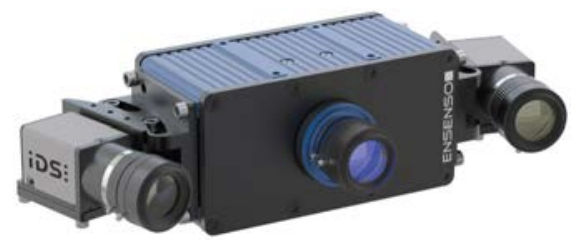

les précédents modèles. L'assistant de configuration est une nouvelle fonction qui vous permet de régler la focale de votre caméra $3 \mathrm{D}$ et de la calibrer.

C'est si simple! Vous pouvez utiliser le sélecteur de caméra Ensenso 4, comparé à la précédente mode FlexView des modèles des séries N35. Par ailleurs, la résolution effectif spatiale en $\mathrm{X}$ et $\mathrm{Y}$ est doublée. Et elle est même bien plus fiable quand elle est utilisée pour des surfaces sombres ou réfléchissantes. Vous pouvez utiliser le SDK Ensenso pour le paramétrage et démarrer en vous servant des modèles Ensenso X, et en suivant les mêmes étapes usuelles que pour pour choisir les composants. Entrez tout simplement vos paramètres dans l'outil de configuration en ligne et celui-ci listera les meilleures combinaisons possibles pour votre application.

\section{CONTACT}

IDS Imaging Development Systems https://fr.ids-imaging.com/ Tél.: +33142214704 